ISSN: 1130-3743 - e-ISSN: 2386-5660

DOI: http://dx.doi.org/10.14201/teoredu2016281209223

\title{
DIMENSIONES DE LA INCLUSIÓN DE LAS TIC EN EL CURRÍCULO EDUCATIVO: UNA APROXIMACIÓN TEÓRICA
}

\author{
Levels of integration of ICT in the curriculum: \\ a theoretical approach
}

\section{Dimensions de l'intégration des TIC dans le programme d'études: une approche théorique}

\section{Sergio URUEÑa LóPEZ}

Ronda de la Feria, 15. 49005 Zamora.sergio.urulo@gmail.com

Fecha de recepción: enero de 2016

Fecha de aceptación: marzo de 2016

\section{RESUMEN}

El presente artículo tiene como principal objetivo analizar desde un punto de vista teórico la triple dimensión que supone la inclusión completa de las Tecnologías de la Información y la Comunicación (TIC) en el currículo educativo. La primera de las dimensiones hace referencia a la inclusión de las TIC como herramientas didácticas para el apoyo del ejercicio docente. La segunda alude a su integración como medida de fomento de un uso eficiente de las mismas. Desde la tercera, se sugiere la inclusión de las Tic como objetos de análisis crítico y de conocimiento. Las dos últimas dimensiones señaladas, las más olvidadas aunque no por ello las menos importantes, resultan fundamentales de cara a la promoción de ciudadanos críticos capaces de tomar decisiones razonables e informadas en lo que respecta a la evaluación, diseño, control e implementación de las nuevas tecnologías.

Palabras clave: TIC; educación; educación tecnológica; competencia digital; ethos tecnológico. 


\section{SUMMARY}

The principal aim of this paper is to analyze from a theoretical point of view the triple dimension that implies the full integration of Information and Communications Technologies (ICT) in the curriculum. The first level refers to the incorporation of ICT as didactic tools to support teaching practice. The second one alludes to the incorporation of ICT as a means for promote an efficient use of it. The third one suggests the incorporation of ICT as objects of critical analysis and knowledge. The last two mentioned dimensions -the most unnoticed but certainly not the least importantare essential for promoting critical citizens with the capacity to take reasonable and informed decisions regarding the assessment, design, monitoring and implementation of the new technologies.

Key words: ICT; education; technological education; digital competence; technological ethos.

\section{SOMMAIRE}

L'objectif principal de cet article est d'analyser d'un point de vue théorique la triple dimension qu'implique la pleine intégration des Technologies de l'Information et de la Communication (TIC) dans le programme d'études. Le premier aspect fait référence à l'intégration des TIC comme outils didactiques pour soutenir la pratique de l'enseignement. Le second se rapporte à l'intégration des TIC comme un moyen pour promouvoir l'utilisation efficace de celle-ci. Le troisième aborde l'intégration des TIC comme des objets d'analyse critique et de la connaissance. Les deux dernières points mentionnées -les plus négligés, mais certainement pas les moins importantssont essentiels pour la formation de citoyens critiques avec une capacité de prendre des décisions raisonnables et éclairées en ce qui concerne l'évaluation, la conception, le suivi et la mise en ouvre des nouvelles technologies.

Mots clés: Tic; éducation; éducation technologique; compétence digitale; ethos technologique.

1. INTRODUCCIÓN: HACIA UNA CONCEPCIÓN AMPLIA DE LA INCLUSIÓN DE LAS TIC EN EL CURRÍCULO EDUCATIVO

El uso de las Tecnologías de la Información y la Comunicación (TIC) ha permeado en un breve espacio de tiempo y de manera contundente en múltiples esferas de la vida, transformando con ello la forma de interrelacionarnos con el medio que nos rodea. El ámbito educativo no ha sido ajeno a estos cambios. Las diferentes políticas educativas promulgadas durante los últimos años vienen a servir de caja de resonancia ante esta tendencia de alcance global que alienta a la inclusión de las TIC en el currículo educativo (Oshima y Maramatsu, 2015). 
La producción bibliográfica existente en la actualidad sobre esta tendencia es abundante, pudiéndose distinguir, al menos, dos líneas generales de investigación. En la primera de ellas se hallarían todos aquellos estudios provenientes de las más diversas áreas de conocimiento (sociología, psicología, pedagogía, filosofía, etc.) en los que se examinan las oportunidades que ofrece el uso de las Tic para el ejercicio docente. Ello incluye tanto los trabajos en los que se debate sobre si resulta beneficioso o no educar con nuevas tecnologías (Ornellas y Sancho, 2015; Almenara, 2007) como aquellos en los que, partiendo de una visión optimista, se ofrecen algunas pautas metodológico-didácticas basadas en ellas (Marshall y Taylor, 2015; Manso et al., 2011; Jaramillo, Castañeda y Pimienta, 2009; Segura, 2007b; Sutherland et al., 2004). Por otro lado, en la segunda de las líneas de investigación se enmarcarían todos aquellos estudios que abordan la relación Tic-educación centrándose en lo que respecta a la adquisición por parte del alumnado de las habilidades y de los conocimientos necesarios para el correcto y eficaz manejo de las nuevas tecnologías (Gisbert y Esteve, 2011; Segura, 2007a; Hawkins, 2002; Hopenhayn, 2002).

En el presente trabajo se mostrará como ambos niveles de análisis («educar con" y "educar para" las TIC), aun cuando puedan estimarse necesarios y convenientes, no resultan de por sí suficientes a la hora de proporcionar un tratamiento completo de la integración de las nuevas tecnologías en el ámbito educativo. Es preciso, en este sentido, complementar ambas dimensiones añadiendo otro nivel hasta la fecha no lo suficientemente tratado y desarrollado, a saber, aquel que hace referencia a la introducción de las nuevas tecnologías como objetos de análisis crítico y de conocimiento. El desarrollo de esta tercera perspectiva, en conjunción con las anteriores, vendría a proporcionar la inclusión completa de las Tic en el currículo formativo.

De este modo, es posible distinguir desde un punto de vista teórico tres dimensiones bajo las que cabría introducir las Tic en la educación: (1) como herramientas para el apoyo y ejercicio de la docencia, (2) como medida de fomento de un uso eficiente de las mismas y (3) como objetos de análisis crítico y de conocimiento. Las dos últimas dimensiones señaladas, justamente las más olvidadas aunque no por ello las menos importantes, circunscriben o incluso amplían la denominada aptitud en el tratamiento de la información y la competencia digital. A cada uno de estos niveles le será dedicado a continuación un epígrafe.

Como se verá, únicamente si concebimos la relación «educación-nuevas tecnologías» desde este enfoque amplio, estaremos en condición de construir una educación que propicie la emergencia de una cultura y ethos tecnológico capaz de impulsar al alumnado a posicionarse como agentes tecnológicos críticos, activos y responsables a la hora de hacer uso, implementar o incluso diseñar las nuevas tecnologías (Quintanilla, 2005, 233-274). No obstante, queda abierta la determinación de la(s) metodología(s) pedagógico-didáctica(s) concreta(s) a seguir de cara a la consecución práctica de la inclusión completa de las Tic a nivel de aula. 


\section{LAS TIC COMO HERRAMIENTAS PARA EL APOYO DE LA DOCENCIA: EDUCAR CON NUEVAS TECNOLOGÍAS}

Desde la primera de las tres dimensiones anteriormente señaladas, las TIC son presentadas atendiendo únicamente a su faceta instrumental como herramientas de apoyo para el ejercicio docente y el proceso de enseñanza-aprendizaje. La cuestión principal que desde aquí se plantea es si las nuevas tecnologías favorecen la dinámica educativa y, de ser así, qué criterios pedagógico-didácticos deben seguirse para aprovechar todas las posibilidades que éstas nos ofrecen.

En general, la percepción del profesorado y del alumnado ante la inclusión de las TIC como herramientas educativas es positiva (Comisión Europea, 2013, 121-128). No obstante, la integración de las tecnologías en la educación no ha resultado ser en el pasado ni resulta ser en la actualidad un proceso simple. La Unión Europea, por ejemplo, ha venido alentando a sus Estados miembros a que hagan uso de las nuevas tecnologías en el ámbito educativo desde mediados de los años 90 (Comisión de las Comunidades Europeas, 1993, 2005, 2007; Bangemann, 1994) y, sin embargo, aún hoy se observan dificultades para que este esfuerzo se traduzca en un uso intensivo y homogéneo de las Tic por parte del profesorado y de los estudiantes (Comisión Europea, 2013, 155). El caso concreto de España ilustra bastante bien la situación a la que se está haciendo referencia. El número de recursos electrónicos disponibles en los centros públicos españoles dedicados a la enseñanza no universitaria ha aumentado rápidamente desde el año 2000, siendo el índice de variación del curso 2002/2003 al 2011/2012 del 418.75\% para el número de ordenadores por estudiante destinado a tareas de enseñanza-aprendizaje, del $424.32 \%$ para el número de ordenadores por estudiante destinado a la docencia y del 390.91\% para el número de ordenadores disponibles por profesor (Instituto Nacional de Estadística, 2012). A pesar de todo este esfuerzo infraestructural que se prolonga hasta nuestro presente y que ha llevado a situar a España en la cabeza de Europa en lo que se refiere a la introducción material de las Tic en el aula, lo cierto es que el uso de las mismas no es el esperado:

A pesar de las buenas infraestructuras disponibles en las aulas, el uso de las mismas en nuestro país no es tan intensivo. Como ejemplo en Educación Secundaria solo el $52 \%$ de los alumnos utiliza ordenadores portátiles o de sobremesa de la escuela al menos una vez a la semana, un punto porcentual por debajo de la media europea. Mientras en bachillerato este porcentaje disminuye al $50 \%$ y en formación profesional asciende al 66\% (Fundación Telefónica, 2015, 48).

Son precisamente estos desequilibrios entre esfuerzos y resultados, entre los medios disponibles y su uso real los que nos hacen recordar que para que exista éxito a la hora de incluir las TIC como recurso metodológico-didáctico es necesario tener más factores y variables en cuenta que los puramente infraestructurales. Segura $(2007 b, 17)$ ya señaló la existencia de tres barreras fundamentales que impiden el uso intensivo de las nuevas tecnologías en el aula: (i) el conocimiento y las habilidades tecnológicas que posee el profesorado, (ii) la gestión que se realiza de 
los recursos en los centros educativos (insuficiente calidad de los recursos, escaso mantenimiento/actualización, inadecuación a las didácticas específicas llevadas a cabo por cada profesor, elevada ratio de alumnos por aula, impertinencia para el contexto sociocultural, etc.) y (iii) las exigencias marcadas por el sistema educativo (sobrecarga de contenidos, rigidez de los criterios de evaluación, etc.).

Y es que a pesar de que las TIC "pueden favorecer la motivación, el interés por la materia, la creatividad, la imaginación y los métodos de comunicación, así como mejorar la capacidad para resolver problemas y el trabajo en grupo, reforzar la autoestima y permitir mayor autonomía de aprendizaje» (Segura, 2007b, 10), ciertamente cabe apaciguar este optimismo recordando que el resultado de la aplicación de las nuevas tecnologías en el ámbito educativo depende en muy alta medida de factores contextuales, del uso que se haga de ellas y de los conocimientos pedagógico-didácticos específicos que se posean sobre su aplicación ${ }^{1}$. La calidad de un profesor por el simple hecho de utilizar recursos tecnológicos no se tornará nunca positiva si éste carece de las habilidades propias de su profesión, esto es, si no sabe desenvolverse adecuadamente en el aula creando un clima propicio para el aprendizaje. Tampoco las tecnologías serán de gran ayuda si no se tienen previamente las aptitudes didácticas específicas que demanda cada tecnología en cuestión. Si bien puede resultar demasiado obvio afirmar a las alturas de nuestro tiempo que educar con nuevas tecnologías requiere más habilidades y conocimientos que los estrictamente necesarios para hacerlas funcionar (Llorente, 2008; García-Varcárcel y Tejedor, 2005), lo cierto es que hasta hace bien poco ello no parecía estar del todo claro. Más bien se pensaba que si un profesor adquiría las competencias técnicas básicas necesarias para el manejo de las Tic, éste podría aplicarlas en la práctica educativa de manera inmediata y efectiva. Una idea equivocada que llevó a centros de formación, universidades, etc., a ofrecer una gran cantidad de cursos formativos destinados exclusivamente a desarrollar las habilidades tecnológicas a nivel usuario del profesorado (Sancho et al., 2008, 15-18).

Hoy sabemos que la educación y la enseñanza son prácticas intencionalmente orientadas hacia un objetivo general concreto -el aprendizaje significativoy que este objetivo modula, por su especificidad y complejidad, las formas de uso que cotidianamente hacemos de los instrumentos, demandando destrezas y conocimientos específicos ${ }^{2}$. De ahí que los esfuerzos de alfabetización dirigidos hacia el profesorado, de darse, deben orientarse hacia la especificidad del proceso de enseñanza-aprendizaje que requieren ciertas TIC enmarcado en su especialidad de enseñanza y no tanto en el aprendizaje de su uso a nivel de

1. Un claro referente del análisis crítico dirigido en contra del optimismo que generalmente rodea al empleo de las nuevas tecnologías en el ámbito educativo es la obra Distrusting Educational Technology: Critical Questions for Changing Times (2014), de Neil SELWYN.

2. Tal y como ha expuesto Quintanilla (1998), entre los elementos constituyentes de los sistemas técnicos se encuentran los objetivos con los que éstos son usados así como los objetivos que han guiado su diseño (los cuales no siempre han de coincidir). 
usuario (aunque esto último sea condición necesaria para que se dé lo primero). Este conjunto de conocimientos didáctico-pedagógicos específicos que exigen las TIC para que resulten un recurso provechoso en el ámbito educativo ha generado una nueva denominación: las Tecnologías para el Aprendizaje y el Conocimiento (TAC) (Enríquez, 2013; Lozano, 2011; Espuny et al., 2010; Sancho, 2008). Transitar de las TIC a las TAC implica pasar de usar hardware y software para enseñar, a enseñar con hardware y software educativo conforme a unos criterios y principios metodológicos claramente trazados.

Todo este complejo entramado de variables que envuelve el uso de las TIC como herramientas para el apoyo de la docencia invita a reflexionar sobre si la elección de enseñar o no con nuevas tecnologías debiera ser, al fin y al cabo, de carácter personal. Cada profesor debe autoevaluar sus posibilidades, conocimientos y habilidades observando a su vez las prioridades y circunstancias de su alumnado para, a partir de ahí, tomar una decisión que vele siempre por el bien de éstos.

\section{LA INCLUSIÓN DE LAS TIC COMO MEDIDA PARA EL FOMENTO DE SU USO EFICIENTE: EDUCAR PARA LAS TIC}

Aun cuando la mayor parte de la bibliografía existente sobre la temática "TICeducación" se centra en la anterior dimensión abordada, las iniciativas en materia de política educativa llevadas a cabo en Europa a mediados de los años 90 tenían entre sus objetivos principales que las Tic se integrasen en el currículo más bien en el sentido que se resalta en este nuevo nivel, a saber, el que hace referencia a la inclusión de las TIC como medida para el fomento de un uso eficiente de las mismas.

La llegada de la sociedad de la información (Castells, 2000) -hoy también sociedad del conocimiento- promovió que la formación enfocada al aprendizaje del uso eficiente de las TIC se convirtiese en un asunto de gran prioridad. Los sistemas educativos, como no podía ser de otro modo, fueron vistos como los principales catalizadores sobre los que era necesario actuar en aras de acomodar a la sociedad a las nuevas exigencias de competitividad que marcaba el mercado:

Debe ponerse gran empeño en garantizar una amplia aceptación pública y un uso real de la nueva tecnología. Preparar a los europeos para el advenimiento de la sociedad de la información es una tarea prioritaria. La educación, la formación y la promoción desempeñarán necesariamente un papel fundamental. [...] A fin de aumentar la concienciación, deberán fomentarse iniciativas regionales y locales, ya sean públicas o privadas (Bangemann, 1994, 5).

La necesidad de que todo ciudadano sepa desenvolverse en esta nuestra sociedad de la información y del conocimiento trajo consigo nuevas metas educativas que vienen a subrayar la urgencia de potenciar el desarrollo de, entre muchas otras capacidades, la competencia tecnológica e informacional o, como se ha venido a 
llamar en España desde el Real Decreto 1513/2006 de 7 de diciembre, la aptitud en el «Tratamiento de la información y competencia digital». Esta competencia tecnológica e informacional pretende desarrollarse en dos sentidos:

1. El primero de ellos hace referencia a la competencia tecnológica en tanto aptitud y capacidad para el uso efectivo de las tecnologías. La presencia cada vez más generalizada de las Tic en todo ámbito hace que una de las tareas del sistema educativo sea la de asegurar que todo alumno disponga de las destrezas tecnológicas necesarias para hacer uso efectivo de las mismas.

2. El segundo de los sentidos alude a la aptitud informacional, esto es, al conjunto de habilidades orientadas a la gestión y la generación de la información (lo que incluye procesos de análisis, organización, síntesis, interpretación, etc.).

Ninguna de estas dos facetas ha de der obviada, pues ambas se encuentran estrechamente interrelacionadas. Favorecer uno de los dos sentidos sin tener en cuenta el otro podría dar lugar a consecuencias no deseadas. Podría pensarse, por ejemplo, que dado que todos los alumnos pueden ser considerados en la actualidad nativos digitales, la problemática relativa al fomento de la adquisición de las aptitudes para el manejo del software y hardware tecnológico resulta en cierto modo superflua. Pero ello no haría más que perpetuar o fomentar la denominada brecha digital (Alva de la Selva, 2015; Quicios, Ortega y Trillo, 2015). No todos los alumnos conviven bajo unas circunstancias que les permitan desarrollar este tipo de aptitudes de una forma adecuada, de ahí que el sistema educativo tenga que poner especial atención en el desarrollo de éstas. Uno de los objetivos de todo sistema educativo es precisamente el de asegurar que todo alumno -especialmente al finalizar la educación obligatoria- se halla en posesión de las habilidades suficientes y necesarias para desenvolverse de manera óptima en la sociedad. Y ello debe cumplirse en lo que respecta a las nuevas tecnologías independientemente de las condiciones materiales de acceso y del nivel de apropiación tecnológica que pueda existir en la ciudad o centro en cuestión en el que se sitúe cada práctica educativa. De la misma manera, dejar de lado el tratamiento de la información puede dirigirnos hacia una situación tan ridícula como que un alumno, poseyendo las habilidades y condiciones materiales para el acceso a la información, no sepa cómo buscarla, procesarla, sintetizarla y/o comunicarla. Las Tic, y en especial Internet, permiten el acceso a gran cantidad de información en cuestión de segundos, casi de manera instantánea. Toda esa información, no obstante, si no es analizada y organizada no puede convertirse en conocimiento, cuanto menos permitir al alumnado lograr un aprendizaje significativo.

La aptitud en el tratamiento de la información debe estar, por ello, necesariamente ligada a la competencia tecnológica o digital si no queremos caer en uno de los dos errores anteriormente señalados. Y es que el medio puede transformar el mensaje, de ahí que cada cual requiera competencias específicas para el tratamiento de la información que transmite. En concreto, las nuevas tecnologías 
-y en especial, la Web 2.0- poseen un modelo hipertextual de organización de la información que se caracteriza por su multisecuencialidad, algo que permite una comunicación interactiva en formato de red (Clement, 1995). Una red que une sus nodos gracias al fenómeno del hipervínculo. Saber gestionar la información provista por las TiC requiere de conocimientos específicos sobre cómo navegar a través de esos hipervínculos sin perdernos en ellos.

Otro de los problemas a los que cabe hacer frente es el de la fiabilidad de los contenidos presentes en la Red. Antes de la llegada de las TIC, si un estudiante quería conseguir información lo más probable es que acudiese a la biblioteca y/o leyera algún libro o revista especializada. Hoy en día eso no ocurre con tanta frecuencia y es muy habitual -sobre todo en los niveles de Educación Secundaria y Bachillerato- que los alumnos consulten Internet para obtener, ampliar y/o cotejar información. El problema reside en que mientras las fuentes clásicas avalan la fiabilidad de la información que contienen (dados los filtros establecidos con anterioridad a la publicación), ese certificado de calidad se asegura en la Web en muy pocas ocasiones. La Red es un maremágnum informacional cuyo rigor puede ser puesto enseguida en duda dada la facilidad con la que pueden crearse y modificarse los contenidos. De ahí que sean los propios usuarios quienes deban realizar ante este tipo de medio un filtrado básico que les permita discriminar la verosimilitud y la calidad de la información a la que acceden.

Asimismo, ha de tenerse en cuenta que no toda la información que recibimos procede de un proceso de búsqueda intencionado y consciente. El abuso que las nuevas generaciones tienden a hacer de las TIC (INJUVE, 2007) promueve que el total de la información que reciben los alumnos fuera del centro y de manera inconsciente sea mucho mayor que la que reciben dentro del mismo y de forma consciente. En total, lo cierto es que estamos expuestos a mucha más información de la asimilable, algo que -según afirman algunos autores- podría provocar el síndrome de la infoxicación, la sobrecarga informativa (Cornella, 2000). Esta sobrecarga informativa, potenciada en parte por el formato bipertextual, podría limitar, en caso de que no se poseyesen las aptitudes adecuadas para gestionar la información, nuestra capacidad de comprensión y de generación de conocimiento (Caldevilla, 2013; Ballesteros y Jiménez, 2012). Precisamente el síndrome de la infoxicación se hace visible cuando un individuo accede y procesa mucha información pero lo hace de forma fragmentada, dando pequeños saltos entre ítem e ítem sin ver las interrelaciones existentes entre ellos y sin profundizar en las peculiaridades de ninguno en concreto.

Todos estos desafíos han ayudado a la transformación del rol del profesorado (González, 2014). Se ha pasado de concebir al profesor como una figura que proporciona información a plantear su actividad como la de un guía que orienta al alumnado a la hora de adquirir las aptitudes básicas marcadas por el sistema educativo. Si antes era el profesorado el que ofrecía el material para el estudio, la nueva pedagogía apunta a que este colectivo debe centrar su actividad en ayudar a sus alumnos a desarrollar las habilidades instrumentales-cognitivas necesarias 
para seleccionar, organizar, analizar, contextualizar y/o sintetizar la información. El objetivo de todo ello es que los estudiantes, a partir de ahí, puedan interpretar y asimilar por sí mismos cualquier paquete informacional y transformarlo en conocimiento. Frente al culto por los objetivos y contenidos conceptuales que se realizaba desde la pedagogía tradicional, la "nueva» fórmula del aprender a aprender invita a otorgar especial protagonismo a los objetivos y contenidos procedimentales y actitudinales.

La aptitud en el tratamiento de la información y la competencia digital precisamente lo que demandan es fomentar determinadas actitudes y promover ciertos procedimientos a la hora de hacer uso de las tecnologías en general y de las TIC en particular. Asegurar un uso eficiente de las nuevas tecnologías y el buen tratamiento de la información requiere, en definitiva, cultivar y promover procedimientos que potencien el desarrollo de habilidades y actitudes que ayuden al tratamiento crítico de la información.

Para ayudar al alumnado a lograr la aptitud en el tratamiento de la información y competencia digital es posible utilizar dos herramientas de sobra estudiadas y reconocidas. Entre sus virtudes, encontramos la efectividad y la fácil adaptabilidad a los objetivos y contenidos concretos fijados para cada sesión o unidad didáctica: se trata de las WebQuests (Catala, Guerrero y Hernández-San-Miguel, 2015) y los Cazatesoros (Adell, 2003). Las WebQuests son «una actividad orientada a la indagación en la que parte o la totalidad de la información con la que los alumnos interactúan proviene de recursos de Internet» (Dodge, 1997). En ella, el estudiante debe solucionar una tarea partiendo de la información obtenida de una serie de fuentes digitales previamente delimitadas a modo de guía por el profesor. Los Cazatesoros, por su parte, son actividades cuya dinámica consiste en plantear una serie de preguntas de complejidad gradual creciente y proporcionar una serie de direcciones webs de las que puede extraerse con mayor o menor nivel de inferencia las respuestas a las mismas (aun cuando existe libertad para consultar otras fuentes). Al final del proceso, el estudiante tiene que hacer frente a una pregunta de mayor dificultad para cuya resolución se requiere haber sintetizado y cribado bien toda la información y haber integrado los conocimientos trabajados en las cuestiones anteriores.

\section{LA INCLUSIÓN DE LAS TIC COMO OBJETOS DE ANÁLISIS CRÍTICO Y DE CONOCIMIENTO: EDUCAR EN LAS TIC}

El desarrollo de la competencia digital circunscribe algunos aspectos a los que raramente se les otorga suficiente atención. Concretamente, convendría tener en cuenta que «la competencia digital incluye utilizar las tecnologías de la información y la comunicación extrayendo su máximo rendimiento a partir de la comprensión de la naturaleza y modo de operar de los sistemas tecnológicos, y del efecto que esos cambios tienen en el mundo personal y sociolaboral, (Real Decreto 1513/2006, Anexo I; énfasis añadido). Es precisamente en la tercera de las dimensiones de 
inclusión de las TIC en el currículo educativo donde se hace hincapié en la necesidad de que éstas sean tomadas, en tanto elementos presentes en el mundo-de-lavida, como objetos de análisis crítico, conocimiento y comprensión.

Las tecnologías en general -y las TIC en particular- son un fenómeno cultural capaz de incidir y transformar el espacio natural y sociocultural del que inicialmente surgieron. La importancia de tomar los sistemas tecnológicos como objetos de análisis crítico, conocimiento y comprensión reside en que sólo percibiendo su raigambre humana (social, política y cultural) podremos orientar la práctica y creación tecnológica hacia fines sostenibles y justos (ya sea en el plano social, económico, político, ecológico, etc.).

Pero, ¿̇en qué sentido un sistema tecnológico puede ser entendido como un fenómeno cultural? Se puede concebir un sistema tecnológico como «una subclase de sistemas técnicos, propios de las sociedades industriales, que se caracterizan por que su diseño y su uso se basan en conocimientos y métodos científicos y en sistemas de valores y procedimientos de evaluación que pueden considerarse racionales" (Quintanilla, 2005, 267). Por ello, "comprender el modo de operar de los sistemas tecnológicos» conlleva tener más conocimientos sobre ellos que los puramente instrumentales, técnicos o científicos. A saber, implica también conocer qué valores y procedimientos de evaluación han motivado la difusión y motivan la perpetuación de ese sistema tecnológico.

Incidir desde el ámbito educativo en la comprensión de esta pluralidad de factores que conforman los sistemas tecnológicos (en especial en lo que respecta a los valores y criterios de evaluación tecnológica) no es una propuesta caprichosa dado el creciente protagonismo que la tecnología ocupa en nuestras vidas. Una de las tareas principales de nuestro presente es la de dotar al alumnado de herramientas que les permitan evaluar, someter a crítica y comprender el modo de operar, los efectos y valores presentes en los sistemas tecnológicos, especialmente de aquellos de los que hacen uso cotidianamente. Es necesario que los alumnos sean capaces de dilucidar los posibles usos de las tecnologías y evaluar sus respectivas consecuencias no con el fin de alentar una ferviente tecnofilia o una insensata tecnofobia, sino de recalcar nuestro papel como agentes tecnológicos y asumir con ello responsabilidades ante el uso, implantación y perpetuación de cualquier tecnología.

Y es que el progreso tecnológico no sigue un proceso de desarrollo autónomo (Quintanilla, 1997). Las tecnologías -y entre ellas las TIC- son desarrolladas e implementadas por personas y, por ello, educar en las nuevas tecnologías implica incidir en el carácter intencional de las mismas e invitar a posicionarnos como sujetos con capacidad de acción y decisión sobre el desarrollo y control de la tecnología (y, de no tenerla, reivindicarla). Los discursos deterministas de la técnica no vienen a hacer ningún bien en este sentido. En ellos, la responsabilidad es despojada del ser humano llevando a la inacción y la parsimonia, esto es, reduciendo las posibilidades de relación del ser humano con las tecnologías a la de «simples consumidores o usuarios» (Quintanilla, 2002, 645). Si no incluimos un enfoque intencional en la lógica interna de los sistemas tecnológicos resulta imposible explicar cuál es el 
papel de los agentes en ellos, algo que limitaría la posibilidad de la participación ciudadana en asuntos de política y gestión tecnológica.

En este sentido, la educación tecnológica se convierte en una propuesta que busca el desarrollo de una ciudadanía (en tanto «el desarrollo tecnológico es un asunto de trascendencia política» (Quintanilla, 2005, 233) que, partiendo de la comprensión del fenómeno tecnológico y sus efectos socioculturales, mantenga una actitud crítica y comprometida con la evaluación del desarrollo tecnológico, demandando su capacidad de autonomía y libertad en el control del mismo. Educar en las TIC significa enseñar a convivir con ellas de una forma responsable y cívica. La inclusión de las TIC en el currículo educativo desde esta tercera dimensión, en definitiva, tendría como objetivos primordiales tanto (i) el fomento de la toma de conciencia de las consecuencias que el uso y diseño de determinadas tecnologías pueden tener en nuestra vida diaria, sociedad y medioambiente, como (ii) la generación de las actitudes y aptitudes necesarias para la realización de propuestas normativas de acción que ayuden a la construcción de una cultura tecnológica adecuada (crítica, justa, razonable y responsable) que plasme o sea representación, en la medida de lo posible, de los intereses de la ciudadanía.

Por todo ello, las TIC pueden y deben tomarse también como objeto crítico de estudio, resultando de especial relevancia el análisis que pueda realizarse en torno a la incidencia y modos de operar de las mismas. En especial, cabe reconsiderar los valores internos que se promueven en servicios digitales de redes sociales como Snapchap, Tinder, Facebook, Twitter, Instagram, etc. El gran uso que hoy se hace de ellos no debe ser ciego: debe estar soportado por una red de conocimientos, valores, actitudes y habilidades procedimentales de corte ético-cívico que permitan tomar conciencia, comprender, evaluar y controlar de forma responsable su funcionamiento.

\section{CONClusiones}

La inclusión que cabe realizar de las Tecnologías de la Información y la Comunicación en el currículo educativo debe ir más allá de la integración de las mismas como instrumentos para el apoyo de la docencia. Educar o no con TIC es una cuestión opcional que debe depender en última instancia del profesorado y de la autoevaluación que ellos mismos realicen sobre sus competencias y aptitudes en el manejo didáctico de las mismas, así como de la idoneidad de su uso en su contexto de trabajo. La verdadera necesidad de la integración de las Tic en el currículo educativo viene dada en otro doble sentido, a saber, por un lado el de (i) lograr las competencias necesarias para el correcto desenvolvimiento en el medio que nos rodea (sociedad tecnológica y del conocimiento) y, por el otro, (ii) el de fomentar una cultura y ethos tecnológico que apoye una ciudadanía crítica y con ímpetu participativo y comprensivo en lo que al uso, diseño e implementación de las tecnologías se refiere. 
(i) La sociedad de la información y del conocimiento precisa de un sistema educativo que respalde la aptitud en el tratamiento de la información y la competencia digital. Desenvolverse con soltura en la era de la información y del conocimiento implica hacer un uso eficiente de las Tic, pues son éstas las que nos facilitan el acceso a una gran cantidad de información. Asimismo, se hace necesario ayudar al alumnado a desarrollar las habilidades instrumental-cognitivas necesarias para la correcta búsqueda, análisis, organización e interpretación de la información existente en la Red. Las materias propias del área humanística (historia, filosofía, etc.) en tanto fomentan de manera natural la capacidad de análisis, síntesis y de reflexión tienen bastante que aportar en este punto. No obstante, debe tenerse en cuenta la especificidad que supone la búsqueda de la información en Internet y, por ello, deben crearse estrategias específicas que aseguren que todo alumno posee las aptitudes necesarias para desenvolverse adecuadamente en el uso de las TiC.

(ii) Por otra parte, la incidencia que las tecnologías tienen en la sociedad y en la vida cotidiana precisa de una educación tecnológica que permita a todo alumno comprender las características de los fenómenos y sistemas tecnológicos. El motivo de ello lo encontramos en que, sólo partiendo de esa comprensión, es posible que éstos se posicionen en su etapa adulta como ciudadanos críticos capaces de tomar decisiones en lo que respecta a la evaluación, diseño, control e implementación de las tecnologías de forma razonada e informada.

Finalmente, cabe mencionar que si bien la inclusión de las nuevas tecnologías en la educación debe ser un elemento a tener en cuenta a la hora de realizar una programación didáctica y de ponerla en práctica, este objetivo no debe monopolizar las metas primordiales del proceso educativo. No se trata de que el proceso de enseñanza-aprendizaje se reduzca a desarrollar la competencia digital en este sentido amplio que se ha venido desarrollando. Consiste más bien en que cada una de las materias (en tanto competencia transversal) aporte su pequeño grano de arena sin que en el proceso pueda verse afectada la consecución de los objetivos específicos fijados en cada una de ellas.

\section{REFERENCIAS BIBLIOGRÁFICAS}

ADELL, J. (2003) Internet en el aula: a la caza del tesoro. Edutec. Revista Electrónica de Tecnología Educativa, 16. Consultado el 14 de julio de 2015. http://edutec.rediris.es/ Revelec2/Revelec16/PDF/Edutec_n16-Adell.pdf.

Almenara, J. C. (2007) Las necesidades de las TIC en el ámbito educativo: oportunidades, riesgos y necesidades. Tecnología y Comunicación Educativas, 21 (45), 5-19.

Alva De la Selva, R. A. (2015) Los nuevos rostros de la desigualdad en el siglo xxi: la brecha digital. Revista Mexicana de Ciencias Políticas y Sociales, 60 (223), 265-285.

Ballesteros, H. y JimÉnez, R. (2012) La fragmentación como universalidad: el miedo a la hiperinformación en la sociedad del conocimiento. Revista Encuentros, 2, 129-138.

Bangemann, M. (1994) Europa y la sociedad global de la información: recomendaciones al Consejo Europeo. Bruselas, Oficina de Publicaciones Oficiales de las Comunidades Europeas. 
DIMENSIONES DE LA INCLUSIÓN DE LAS TIC EN EL CURRÍCULO EDUCATIVO...

Caldevilla, D. C. (2013) Efectos actuales de la 'Sobreinformación' y la 'Infoxicación' a través de la experiencia de las bitácoras y del proyecto I+D avanza 'Radiofriends'. Revista de Comunicación de la SEECI, 30, 34-56.

CASTElls, M. (2000) La era de la información. Madrid, Alianza.

CATAla, C. L.; Guerrero, C. S. y Hernández-SAn-Miguel, J. (2015) Revisión de la producción científica sobre WebQuest en los últimos 20 años: análisis bibliométrico en Scopus y Web of Science. Edutec. Revista Electrónica de Tecnología Educativa, 52. Consultado el 14 de julio de 2015. http://www.edutec.es/revista/index.php/edutec-e/article/ view/603/pdf_47.

ClÉMENT, J. (1995) Du texte à l'hypertexte: vers une épistémologie de la discursivité hypertextuelle, en Balpe, J. P.; Lelu, A. y SAleH I. (eds.) Hypertextes et hypermédias: Réalisations, Outils, Méthodes. Paris, Hermés-Lavoisier.

COMISIÓN DE LAS COMUNIDADES EUROPEAS (1993) Crecimiento, competitividad, empleo: retos $y$ pistas para entrar en el $s$. XXI. Bruselas, Oficina de Publicaciones Oficiales de las Comunidades Europeas.

Comisión de las Comunidades EuRopeas (2005) Propuesta de recomendación del Parlamento Europeo y del Consejo sobre las competencias clave para el aprendizaje permanente. Bruselas, Oficina de Publicaciones Oficiales de las Comunidades Europeas. Consultado el 14 de julio de 2015. http://www.europarl.europa.eu/meetdocs/2004_2009/documents/com/com_com(2005)0548_com_com(2005)0548_es.pdf.

COMISIÓN DE LAS COMUNIDADES EUROPEAS (2007) Competencias clave para el aprendizaje permanente. Un Marco de Referencia Europeo. Bruselas, Oficina de Publicaciones Oficiales de las Comunidades Europeas. Consultado el 14 de julio de 2015. http://eur-lex.europa. $\mathrm{eu} /$ legal-content/ES/TXT/HTML/?uri=URISERV:C11090\&from=ES.

COMISIÓN EUROPEA (2013) Survey of Schools: ICT in Education. Benchmarking Access, Use and Attitudes to Technology in Europe's Schools. Bruselas, Oficina de Publicaciones Oficiales de las Comunidades Europeas. Consultado el 14 de julio de 2015. https://ec.europa.eu/ digital-single-market/sites/digital-agenda/files/KK-31-13-401-EN-N.pdf.

Cornella, A. (2000) Cómo sobrevivir a la infoxicación. Infonomia.com, 8. Consultado el 14 de julio de 2015. http://www.infonomia.com/img/pdf/sobrevivir_infoxicacion.pdf.

Dodge, B. (1997) Some Thoughts About WebQuests. The WebQuest Page. Consultado el 14 de julio de 2015. http://webquest.sdsu.edu/about_webquests.html.

EnRíQueZ, S. C. (2013) Luego de las TIC, las TAC. En II Jornadas Nacionales de TIC e Innovación en el Aula. Consultado el 14 de julio de 2015. http://sedici.unlp.edu.ar/bitstream/ handle/10915/26514/Documento_completo.luego\%20de\%20las\%20Tic,\%20las\%20 TAC\%20(1).pdf?sequence $=1$.

Espuny, C.; Gisbert, M.; GonZÁlez, J. y Coiduras, J. (2010) Los seminarios tac. Un reto de formación para asegurar la dinamización de las TAC en las escuelas. Edutec. Revista Electrónica de Tecnología Educativa, 34. Consultado el 14 de julio de 2015. http://edutec.rediris.es/Revelec2/revelec34/pdf/Edutec-e_n34_Espuny_Gisbert_Gonzalez_Coiduras.pdf.

FundaCión TelEFónica (2015) La sociedad de la información en España 2014. Barcelona, Ariel.

García-Valcárcel, A. y Tejedor, F. J. (2005) Condiciones (actitudes, conocimientos, usos, intereses, necesidades formativas) a tener en cuenta en la formación del profesorado en TIC. Enseñanza \& Teaching, 23, 115-142.

Gisbert, M. y Esteve, F. (2011) Digital Learners: la competencia digital de los estudiantes universitarios. La Cuestión Universitaria, 7, 48-59. 
GonZÁlez, M. M. C. (2014) El rol del profesor en la educación virtual. Revista Iberoamericana para la Investigación y el Desarrollo Educativo, 12. Consultado el 14 de julio de 2015. http://ride.org.mx/1-11/index.php/RIDESECUNDARIO/article/view/830/811.

HAwKIns, R. J. (2002) Ten lessons for ICT and education in the developing world, en KIRKMAN, G.; CORNELIUS, P. K.; SACHS, J. D. y SCHWAB, K. (eds.) The global information technology report 2001-2002: Readiness for the Networked World. New York, Oxford University Press, 38-43.

Hopenhayn, M. (2002) Educar para la sociedad de la información y de la comunicación: una perspectiva latinoamericana. Revista Iberoamericana de Educación, 30, 187-217.

Injuve (2007) Uso de TIC, Ocio y Tiempo libre, Información. Consultado el 14 de julio de 2015. http://www.injuve.es/sites/default/files/Sondeo2007-2b.pdf.

Instituto Nacional de Estadística (2012) Tecnología de la información en la enseñanza no universitaria. Principales resultados. Madrid, INE. Consultado el 14 de julio de 2015. http://www.ine.es/dynt3/inebase/index.htm?type=pcaxis\&path=/t13/ p022/\&file=pcaxis.

Jaramillo, P.; Castañeda, P. y Pimienta, M. (2009) Qué hacer con la tecnología en el aula: inventario de usos de las TIC para aprender y enseñar. Educación y Educadores, 12 (2), 159-179.

Llorente, M. C. (2008) Aspectos fundamentales de la formación del profesorado en TiC. Pixel-Bit: Revista de Medios y Educación, 31, 121-130.

LozANO, R. (2011) De las TIC a las TAC: tecnologías del aprendizaje y el conocimiento. Anuario ThinkEPI, 5, 45-47.

Manso, M.; Pérez, P.; Libedinsky, M.; Light, D. y Garzón, M. (2011) Las tic en las aulas. Experiencias latinoamericanas. Buenos Aires, Paidós.

MARSHALL, S. y TAYLOR, W. (eds.) (2015) ICT in education: Innovation, implementation, perceptions and experiences. International Journal of Education and Development using Information and Communication Technology, 11 (3).

Ornellas, A. y SANCho, J. (2015) Three Decades of Digital ICT in Education: Deconstructing Myths and Highlighting Realities, en Harmes, M. K.; Hujjser, H. y Danaher, P. A. (eds.) Myths in Education, Learning and Teaching: Policies, Practices and Principles. New York, Palgrave Macmillan, 135-150.

Oshima, K. y Muramatsu, Y. (2015) Current Situation and Issues Related to ICT Utilization in Primary and Secondary Education. Fujitsu Scientific \& Technical Journal, 51 (1), 3-8. Consultado el 14 de julio de 2015. http://www.fujitsu.com/downloads/MaG/vol51-1/ paper01.pdf.

Quicios, M. P.; Ortega, I. y Trillo, M. P. (2015) Aprendizaje ubicuo de los nuevos aprendices y brecha digital formativa. Pixel-Bit. Revista de Medios y Educación, 46, 155-166.

Quintanilla, M. Á. (1997) El concepto de progreso tecnológico. Arbor, 157 (620), 377-390.

Quintanilla, M. Á. (1998) Técnica y Cultura. Teorema: Revista Internacional de Filosofía, 8 (3), 49-69.

Quintanilla, M. Á. (2002) La democracia tecnológica. Arbor, 173 (683-684), 637-651.

Quintanilla, M. Á. (2005) Tecnología: un enfoque filosófico y otros ensayos de filosofía de la tecnología. México, Fondo de Cultura Económica.

SANChO, J. M. (2008) De TIC a TAC, el difícil tránsito de una vocal. Investigación en la Escuela, 64, 19-30.

Sancho, J. M.; Ornellas, A.; Sánchez, J. A.; Alonso, C. y Bosco, A. (2008) La formación del profesorado en el uso educativo de las Tic: una aproximación desde la política educativa. Praxis Educativa, 12, 10-22. 
Segura, M. (2007a) Acciones institucionales y programas para la integración de las Tic, en MinISTERIO DE EDUCACIÓN Y CIENCIA (ed.) Introducción temprana a las TIC: Estrategias para educar en un uso responsable en educación infantil y primaria. Madrid, Secretaría General de Educación, 9-24.

SEgura, M. (2007b) Las Tic en la educación: panorama internacional y situación española, en Fundación Santillana (ed.) Las Tecnologias de la Información y la Comunicación (TIC) en la educación: retos y posibilidades. Madrid, Fundación Santillana, 11-49. Consultado el 14 de julio de 2015. http://www.fundacionsantillana.com/upload/ficheros/ paginas/200906/xxii_semana_monografica.pdf.

SELWYN, N. (2014) Distrusting Educational Technology: Critical Questions for Changing Times. New York, Routledge.

Sutherland, R.; Armstrong, V.; Barnes, S.; Brawn, R.; Breeze, N.; Gall, M.; Matthewman, S.; Olivero, F.; TAYlor, A.; Triggs, P.; Wishart, J. y John, P. (2004) Transforming teaching and learning: embedding ICT into everyday classroom practices. Journal of Computer Assisted Learning, 20 (6), 413-425. 